\section{¿Cómo curar al sistema de salud argentino?}

\author{
Federico Tobar $^{1}$
}

"No duden que un grupo de personas inspiradas puedan cambiar las cosas. De hecho, eso es lo que ha ocurrido desde el comienzo de la historia"

Margaret Mead

Palabras clave: sistemas de salud, Argentina, seguros de salud.

\footnotetext{
1 Jefe de gabinete del Ministerio de Salud de Argentina, Buenos Aires.
}

En 1977, Ilya Prigogine se hizo acreedor del Premio Nobel de Química por sus estudios sobre los fenómenos aleatorios. Este físico y químico belga nacido en Rusia demostró que, llevado a condiciones extremas, un sistema en equilibrio inestable puede alcanzar un equilibrio muy superior al inicial. Desde entonces Prigogine se hizo tan conocido entre los científicos sociales como entre los especialistas en ciencias exactas. ¿Por qué? Tal vez porque sus hallazgos constituyen la prueba científica de que vale la pena mantener la ilusión. Demuestran que, como afirma la sabiduría popular, cuando se tocó fondo se vuelve a subir más rápido.

$\mathrm{Al}$ igual que el país, el sistema de salud argentino no está condenado al fracaso. Es posible que las condiciones actuales de extrema precariedad sirvan para estimular una transformación, una reforma hacia un equilibrio mucho más estable. El motivo es sencillo. Mientras restan algunos recursos, se da lugar a un juego de intereses que lleva a un equilibrio cada vez más precario. Solo cuando se han agotado prácticamente todos los recursos aparece la preocupación por la salud de todos como un asunto más importante que los intereses particulares.

Un ejemplo concreto es la construcción de la "canasta básica" de prestaciones cubiertas por la seguridad social, que en Argentina hemos denominado Programa Médico Obligatorio (PMO). Mientras la seguridad social contaba con recursos, resultaba muy difícil establecer un ordenamiento restrictivo. Cada grupo de prestadores ejercía su influencia para que los servicios que brindaba no fueran excluidos. El resultado era un menú de prestaciones tan ambicioso como insostenible con los recursos financieros disponibles en el sector. Cuando la cadena de pagos del sector estalló y los servicios fueron definitivamente interrumpidos, la gran mayoría de las obras sociales no pudieron garantizar a sus beneficiarios el acceso a los bienes y servicios establecidos en el PMO, e incluso gran parte de las empresas de medicina prepagada (EMP) se encontraron en condiciones similares. En ese contexto extremo puede aparecer un nuevo PMO de emergencia que configure un modelo de prestaciones mucho más racional y sostenible que el anterior.

Crisis y oportunidad están juntas, como indica el tantas veces citado ideograma chino que se vale de los mismos trazos para aludir a la crisis y a la oportunidad. La situación sanitaria depende, en parte, de cómo se mire. Muchas son las señales alentadoras que indican que el sector de la salud puede alcanzar 
un equilibrio más estable, que nuestro sistema puede renacer más fuerte y saludable. Por ejemplo, de repente nos volvimos más eficientes. Si lo medimos en valores capitados, nuestro gasto en salud se redujo drásticamente, pasando de US\$ 612 en 2001 a \$183 proyectados para 2002. Ya no somos el país de América Latina que más invierte en salud. Primero cayó el gasto en pesos (casi dos mil millones entre 2000 y 2001), y a esto se agregó la devaluación del peso con relación al dólar con la caída de la convertibilidad. Algo similar ocurrió con el precio de los medicamentos. De un sacudón pasaron a costar la mitad en dólares y dejaron de ser los más caros del continente.

\section{LA ENFERMEDAD DEL SECTOR DE LA SALUD}

Argentina es un país avanzado en su transición epidemiológica, que ha sido pionero en la erradicación de algunas enfermedades infecciosas y en el desarrollo de una respuesta social organizada a los problemas de salud de su población. Sin embargo, la grave situación económica actual, sumada al progresivo deterioro de las condiciones sociales, compromete la sostenibilidad del sistema y sus servicios, y amenaza con deteriorar las condiciones de salud de la población. De no implementarse soluciones rápidas y eficaces, la población argentina podría involucionar varios años en lo que respecta a su situación de salud.

La salud de los argentinos está amenazada. La cobertura de los seguros de salud tiende a disminuir. Los recursos de las obras sociales se han contraído y ello repercutió en reducciones y atrasos en los pagos a los prestadores. La cobertura de vacunas ha retrocedido y en los hospitales públicos comienzan a faltar insumos. En síntesis, el sistema está enfermo, ya ha ingresado en la sala de urgencias y hace falta intervenir rápidamente. Hay que salvar a los trabajadores de la salud, que son nuestro principal recurso sanitario. Hay que salvar los establecimientos de salud públicos y privados. Hay que salvar nuestras obras sociales.

Por este motivo debe pensarse en el sector de la salud argentino en, al menos, dos fases. Por un lado debemos obtener una propuesta para salir de la situación de emergencia del sector. Por otro lado, debemos avanzar hacia una reforma estructural que permita prevenir futuras crisis.

El Ministerio de Salud ya está desarrollando un activo Programa de Emergencia Sanitaria cuyos objetivos centrales son: a) garantizar el acceso a los bienes y servicios de salud básicos para la población argentina, y b) reducir y controlar los riesgos sanitarios y epidemiológicos. Sus prioridades son, en primer lugar, reactivar los servicios públicos de salud. Para ello se garantizó la provisión de medi- camentos e insumos a los hospitales. Esto permitirá que todos los argentinos que necesiten tratamiento hospitalario puedan recibirlo de forma adecuada.

Para ello se consolidó con recursos del gobierno nacional y donaciones un fondo que permite adquirir medicamentos e insumos estratégicos para ser distribuidos entre los hospitales polivalentes de las provincias. En el ámbito del Consejo Federal de Salud, integrado por todos los ministros provinciales y coordinado por el Ministerio Nacional, se acordó una fórmula precisa y transparente para definir la asignación de recursos que corresponde a cada jurisdicción.

En segundo lugar, el Programa de Emergencia buscó garantizar la continuidad de los programas prioritarios, como el maternoinfantil y todos los relacionados con enfermedades transmisibles (tuberculosis, enfermedad de Chagas, dengue, fiebre amarilla, paludismo, sida y enfermedades de transmisión sexual).

Además se está implementando una política innovadora para que todo el mundo tenga acceso a los medicamentos de uso ambulatorio. Por un lado, se lleva a cabo a través de un seguro de medicamentos que permitirá a los desempleados y a sus familias acceder a los medicamentos directamente en las farmacias. Por otro lado, se va a proveer de medicamentos a los Centros de Atención Primaria de la Salud (CAPS), para que todos los que acudan a una consulta médica puedan volver a su casa con el medicamento adecuado, sin tener que pagar nada por ello.

\section{DE LA TERAPIA INTENSIVA A LA REHABILITACIÓN}

El sistema de salud está enfermo, pero se puede curar y la recuperación puede ser mayor y más definitiva que unos años atrás, cuando ni siquiera vislumbrábamos esta crisis. Hoy los actores del sector de la salud estamos más cerca de ponernos de acuerdo, establecer prioridades y articular esfuerzos. El tratamiento adecuado para curar al sistema es "definir prioridades" y "articular intereses y acciones". La experiencia mundial indica que ese es el tratamiento adecuado para un diagnóstico conocido: la sobreexposición a políticas tóxicas y la atrofia del sistema de gestión.

Pero no nos podemos limitar a paliar los efectos de la crisis; también debemos reformar el sistema para poder tener más salud con los recursos disponibles. ${ }^{2}$ Lo que necesitamos es adoptar mejores políticas que, en primer lugar, nos den más años y calidad de vida, no palabras vacías. En segundo

\footnotetext{
2 Ya no podemos proclamar "más salud por el mismo dinero" porque los recursos del sector se han contraído de forma significativa.
} 
lugar, políticas que curen al sistema de salud, que erradiquen sus males. Esto implica:

- Formular políticas saludables. Hace falta un Plan Nacional de Salud que integre todos los subsistemas, que establezca prioridades claras y precisas, con objetivos concretos, y que articule las acciones de todas las jurisdicciones.

- Rescatar las funciones rectoras en salud. Recuperar la misión del Ministerio de Salud de la Nación como el gran director de orquesta que defina las prioridades sanitarias de los argentinos. Porque ya no podemos continuar dilapidando recursos, duplicando funciones y acciones en diferentes lugares. Es preciso sincronizar esfuerzos, no solamente en el gobierno nacional sino también colaborando, orientando y fortaleciendo a los ministerios provinciales, es decir, rescatando también la misión del Consejo Federal de Salud.

- Establecer una adecuada división de responsabilidades en el sistema de salud. Esto significa avanzar hacia un sistema plural. Aunque un modelo único, como el Servicio Nacional de Salud británico o el Sistema Único de Salud brasileño, podría parecer una alternativa más eficaz y equitativa, hoy no representa una solución realista ni viable para Argentina. La reconstrucción del modelo argentino de salud debe seguir el precepto de primum non nocere, evitando destruir o dañar lo que ha restado de nuestros antiguos servicios de salud. Necesitamos definir un papel para cada uno, para los hospitales públicos y privados, para las obras sociales y las EMP y para los tres niveles de gobierno (nacional, provincial y municipal), buscando que el todo sea más que la suma de las partes y no una dispersión de esfuerzos y recursos.

\section{UNA PROPUESTA PARA LA REORGANIZACIÓN DEL SISTEMA}

Este sistema de salud más saludable podría operar sobre tres grandes ejes:

I. Un mercado de seguros de salud que opere en condiciones de competencia regulada.

II. La universalización de la cobertura médica a través de seguros públicos de salud.

III. Un despliegue de acciones localizadas de atención primaria, promoción y prevención.

\section{Un mercado de seguros de salud que opere en condiciones de competencia regulada}

El elenco argentino de aseguradores de salud es muy grande y variado. Operan más de doscien- tas obras sociales nacionales, algunas de origen sindical y otras estatales, una cantidad similar de EMP y un número aun mayor de mutuales. En la gran mayoría de los casos estos aseguradores actúan como financiadores que compran servicios en un mercado de prestadores también muy amplio y variado.

La propuesta es que correspondería al nivel central (nacional), a través de la Superintendencia de Servicios de Salud, actuar como el gran director de una orquesta de aseguradores y prestadores, estableciendo incentivos y sanciones para obtener el máximo resultado en términos de niveles de salud. La competencia entre aseguradores no sería por precios ni por servicios cubiertos, y ni siquiera por calidad. Sería por resultados sanitarios obtenidos, es decir, por niveles de salud de la población cubierta. Para ello recibirían una capitación ajustada según el riesgo (el sexo y la edad de los beneficiarios), de manera que se evite la selección adversa. Pero además recibirían incentivos por sus conquistas epidemiológicas, como, por ejemplo, el aumento de la edad media a la que ocurren los infartos, la reducción de las hospitalizaciones por causas evitables y de los accidentes, etc. Esto reorientaría las preocupaciones de los aseguradores hacia la salud y la satisfacción de los usuarios, ya que para que su negocio fuera rentable deberían mejorar los niveles de salud y mantener satisfechos a sus beneficiarios.

Una variable clave para aumentar la competencia sin debilitar el sistema en su conjunto es establecer un menú de prestaciones (el PMO) sostenible. De hecho, a medida que se aumenta la cobertura, se está concentrando el mercado, ya que serán menos los ofertantes que podrán competir. El PMO argentino en sus versiones históricas (decretos 247/1996 y 939/2000) constituía la quintaesencia del sueño argentino: "vivir en un Estado de bienestar a la europea con la disponibilidad de recursos del tercer mundo".

El PMO debe ser revisado y redefinido, no una vez, sino de forma periódica y sobre fundamentos epidemiológicos (incluir la cobertura de las principales enfermedades prevalentes), garantizando su sostenibilidad actuarial (evaluar los riesgos que involucra cada prestación para cada grupo etario) y estableciendo prioridades de inclusión a través de criterios de eficiencia (medir el impacto de la inclusión de cada prestación en términos de carga de morbilidad y costos, para luego ordenarlas según su importancia). ${ }^{3}$

\footnotetext{
3 De acuerdo con lo dictado por el Decreto de Necesidad y Urgencia $\mathrm{N}^{\circ} 486 / 2002$ que establece la Emergencia Sanitaria en Argentina, el Ministerio de Salud formuló un Programa de Prestaciones Esenciales Garantizadas, que representa un avance en ese sentido.
} 
Una segunda variable clave para viabilizar el sistema es organizar y socializar el financiamiento de los tratamientos de enfermedades catastróficas mediante un seguro nacional. Existe un grupo de enfermedades de baja incidencia y alto costo cuyo tratamiento implica gastos tan elevados que desequilibran las ecuaciones presupuestarias de los organismos financiadores. Muchas de ellas cuentan con leyes específicas y sus tratamientos deben ser impartidos de manera obligatoria (discapacidades, diabetes, insuficiencia renal crónica). Frente a la escasez de recursos y a la imposibilidad de responder a la demanda, muchas entidades financiadoras han puesto en práctica distintos mecanismos, tales como la transferencia del riesgo a los prestadores, convirtiéndolos en prefinanciadores del sistema o estableciendo diversas barreras de acceso al tratamiento.

Para revertir esta situación resulta conveniente implementar un seguro nacional que contemple a la totalidad de la población y permita la cobertura de estas enfermedades. La financiación del mismo debería estar integrada por todos los financiadores actuales, con una cuota por determinarse. Los recursos se constituirían en lo que se denomina una reserva técnica, siendo el Estado el garante de dichos fondos, que no podrían ser asignados a otros fines. Las prestaciones, los medicamentos e insumos deberán estar establecidos impositivamente, y las poblaciones perfectamente determinadas, de modo que se puedan aplicar cálculos actuariales.

\section{Universalización de la cobertura médica mediante seguros públicos de salud}

A su vez, los gobiernos provinciales conducirían las reformas en sus provincias según sus posibilidades y necesidades, respetando las particularidades de cada una, pero con una orientación común. Buscarían ampliar la cobertura y el acceso a los servicios médicos para la mayor parte de la población provincial. Esto se podría lograr fortaleciendo su poder de compra, consolidando seguros provinciales de salud centrados en dos cosas: la obra social provincial como asegurador, y los hospitales públicos como principales prestadores.

La emergencia hace más viable esta reforma porque la interrupción de la cadena de pagos y el consecuente corte de servicios en las obras sociales ha unificado el modelo, conduciendo toda la demanda hacia los servicios públicos. Es decir, la situación de emergencia forzó a los servicios públicos a asumir la cobertura de un 70 a $80 \%$ de la población, sin contar con fondos adicionales ni una unificación de fondos, sin contar con apoyo técnico para el diseño de un modelo de prestaciones adecuado, sin identificar a los destinatarios y sin avanzar hacia esquemas de financiación de los servicios públicos que incentiven un mejor desempeño. La propuesta consiste en extender la cobertura, fundamentalmente a los sectores de la población con mayores carencias, unificando el financiamiento a nivel de los estados provinciales y organizando una amplia red de prestaciones integrada por servicios públicos y privados.

Las dudas clave son: ¿en qué medida se puede apoyar el seguro sobre la estructura de la obra social provincial? y ¿qué plan de cobertura se podría sostener con los recursos disponibles en cada jurisdicción? Para dar respuesta adecuada a estas preguntas se contemplan las siguientes definiciones:

Una estrategia gradual para asumir la cobertura de los beneficiarios de la obra social de los jubilados (el Instituto Nacional de Servicios Sociales para Jubilados y Pensionados, más conocido como Pami, en alusión a su Programa de Asistencia Médica Integral). Las provincias que cuenten con una obra social provincial autónoma y equilibrada se harían cargo de los beneficiarios del Pami y, a la larga, del Programa Federal de Salud (Profé), que brinda cobertura a los beneficiarios de pensiones no contributivas, pero aquellas que no presenten las condiciones adecuadas deberían comprometerse con un conjunto de reformas para comenzar a contar con el apoyo técnico y financiero de la nación.

En el ámbito de cada provincia se organizará un fondo de salud. El mismo estará integrado básicamente por tres fuentes: a) recursos del tesoro provincial que hasta ahora han sido destinados a la atención médica, b) recursos de la obra social provincial (aportes y contribuciones), y c) recursos transferidos desde un fondo compensador nacional.

Toda la población provincial que carezca de cobertura de seguros de salud será identificada y empadronada. Se entregará un carné de afiliación a toda la población que no figure en los padrones de beneficiarios de la Administración Nacional de la Seguridad Social (Obras Sociales Nacionales) ni en la lista de beneficiarios de EMP.

El seguro público tendrá la responsabilidad de brindar a sus beneficiarios una cobertura médica que debería aproximarse al PMO. Sus prestadores conformarán una mezcla de servicios públicos de la red provincial y municipal junto a servicios privados.

En la medida en que toda la población estaría cubierta por los diferentes esquemas de seguros de salud, se podría modificar la financiación de los servicios públicos que incorporen incentivos para reducir los costos administrativos y aumentar la productividad de los hospitales públicos. 
Un despliegue de acciones localizadas de prevención y de promoción de la salud

Los municipios, a su vez, concentrarían sus esfuerzos en desplegar una estrategia de Atención Primaria de la Salud (APS), así como en acciones preventivas y de promoción de la salud, desarrollando políticas saludables, informando y modelando conductas. Desde esta propuesta no se impediría que los municipios que estén en condiciones de hacerlo asuman también funciones de asegurador, como es el caso de algunas ciudades de la provincia de Buenos Aires, pero se buscaría que el objetivo de los gobiernos municipales no se limitase a garantizar cobertura, sino a alcanzar progresivamente los rasgos de un municipio saludable. Si los gobiernos provinciales asumen la función aseguradora no es necesario que lo hagan también los municipios. Por otro lado, para lograr economías de escala, un esquema de seguros públicos de salud debería involucrar a un número muy alto de beneficiarios que se acercaría a las 100000 personas.

Es evidente que la estrategia de APS es muy eficiente y también que su implementación adecuada debe ser planteada a nivel local. Sin embargo, hay dos obstáculos históricos para ello en Argentina. Por un lado está el carácter hospitalocéntrico del sistema. En Argentina, los centros de mayor complejidad todavía destinan gran parte de sus servicios al primer nivel de atención. Por otro lado, diseñar, implementar y evaluar un sistema de APS implica un conjunto de costos que muchas veces los municipios no consiguen mantener. Para superar estos obstáculos, el ministerio nacional deberá desarrollar una estrategia que permita asistir a los CAPS que se comprometan a implementar un modelo adecuado de APS. La asistencia desde la nación consistirá, en primer lugar, en el diseño de un modelo de atención adecuado para la APS en centros locales. En segundo lugar, el ministerio nacional se ocupará de la provisión de medicamentos genéricos para garantizar el acceso a ellos por parte de la población de bajos recursos. Esto implica incorporar un incentivo directo sobre la población para dirigir la demanda de servicios de salud hacia los CAPS en lugar de hacia los hospitales. Además, la nación acreditará y clasificará los CAPS, realizará capacitación e introducirá incentivos para los profesionales responsables.

\section{CONCLUSIONES}

Para mejorar la salud de los argentinos hace falta desplegar un esfuerzo articulado y federal. El modelo fragmentado está agotado. Hay que extender la cobertura e igualar las condiciones de acceso.
Es decir, lograr igual respuesta para igual necesidad. Esto requiere un esfuerzo organizado para que los hospitales públicos mejoren y para que las obras sociales no quiebren ni reduzcan sus prestaciones.

Como un gran director de orquesta, el ministerio nacional deberá dirigir, coordinar y articular a los actores del sector para obtener los mejores resultados. Su primera función deberá ser dirigir el cambio del sistema. En segundo lugar, puesto que la mano invisible del mercado no resuelve los problemas de salud de la población, corresponde al ministerio nacional ejercer una fuerte regulación de la oferta y de la demanda de los bienes y servicios de salud. En tercer lugar, para garantizar mejores condiciones de funcionamiento de los seguros públicos, sociales y privados de salud, es necesario que el país organice un seguro nacional para las enfermedades de baja prevalencia y alto costo. En cuarto lugar, el país deberá concentrar sus esfuerzos en monitorear y evaluar estructuras, procesos y resultados de salud entre regiones, provincias y municipios, así como entre los beneficiarios de las distintas obras sociales, y propulsar la forma de reducir las diferencias y alcanzar mayor equidad.

Los gobiernos provinciales deberán organizar sus seguros públicos de salud. Esto implica hacerse cargo de la planificación, monitoreo y financiación de los servicios, pero no de su provisión directa. $\mathrm{Si}$ toda la población de cada jurisdicción cuenta con un carné de cobertura, algunos de EMP, otros de obras sociales nacionales, otros de la obra social provincial y el resto del seguro público provincial, se podrían financiar los servicios públicos a través de la demanda que atienden y de los resultados que obtienen. Esta debería ser la tarea principal de la provincia, ya que la provisión y gestión de los servicios debería ser lo más autónoma y descentralizada que resulte posible.

Los hospitales públicos deben ser de la gente. La única forma de mejorar definitivamente los servicios públicos es poniéndolos en manos de la comunidad organizada. Esto se logrará por tres vías. Por un lado, la conducción deberá ser descentralizada hacia consejos municipales con fuerte participación comunitaria. Por otro lado, los usuarios deberán ser claramente identificados; cada ciudadano debe tener un carné para ser atendido. En tercer lugar, la financiación de los servicios deberá estar vinculada a los resultados perseguidos y logrados.

A su vez, los municipios deberían concentrar sus esfuerzos en definir e implementar políticas en materia de prevención, promoción de la salud y fiscalización: todo aquello que convierte a la salud en un bien público. Si el ministerio nacional fortalece su capacidad de monitoreo y seguimiento epidemiológico, podría ejercer un importante papel en la 
asistencia técnica a los municipios para la implementación de este tipo de políticas.

Para compensar las diferencias regionales se constituiría un fondo compensador manejado por el país e integrado por recursos que hoy son destinados al Pami, al Profé y a parte de los programas verticales del ministerio. Además de garantizar la equidad de la financiación, el fondo permitirá incentivar a los seguros provinciales para obtener mejores resultados sanitarios. De forma similar actuará el fondo que financiará a las obras sociales nacionales, mutuales y EMP que se inscriban como agentes del seguro. El objetivo sería consolidar un gran fondo de salud que sea administrado por el país y que permita una regulación adecuada, tanto de las obras sociales nacionales y provinciales como de las EMP.

Además, se trata de un esfuerzo racionalizador que cambiaría de forma progresiva las conductas de todos los actores involucrados en el sistema de salud, desde el gobierno nacional hasta los propios ciudadanos, pacientes y usuarios. Probablemente de esta forma no se logre resolver de inmediato todos los problemas de nuestro sistema de salud. Sin embargo, cabe recordar aquella fábula que narraba un incendio en el bosque. Cuando todos los animales ya habían huido, el león, al salir, se encuentra con el colibrí que volaba apresurado desde el río hasta el foco del incendio llevando agua en su piquito. El león le dijo:

- Colibrí, vámonos, ya está todo perdido, no hay nada más que hacer.

Puede ser - le respondió el colibrí- pero yo al menos hice mi parte.

\section{SYNOPSIS}

\section{How do we heal the Argentine health care system?}

This article proposes a set of measures to reform the Argentine health care system and turn the country's current crisis into an opportunity for progressive, sustainable change. The proposal consists of a model for the intergovernmental division of health responsibilities. The national government would be responsible for strengthening its leadership role and for developing national insurance for low-prevalence high-cost diseases. With the provincial governments, the insurance role would be strengthened, with public health insurance making certain that there is universal coverage. Public hospitals would function as autonomous entities financed by social insurance, private insurance, and provincial public insurance. Municipalities would have an active role in disease prevention and health promotion, principally through primary care.
BUILDING

STANDARD-BASED

NURSING

INFORMATION

SYSTEMS

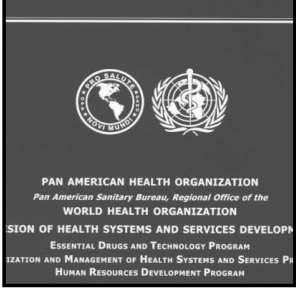

$2001 \cdot 160 \mathrm{pp}$ ISBN 9275123640 Order Code: OP 140

Prices:US\$24.00/

US\$ 18.00 in Latin

America and the Caribbean

\section{Building Standard-based Nursing Information Systems}

Building Standard-based Nursing Information Systems is directed to practicing and student nurses, health care professionals involved in the implementation of information systems, and information technology professionals working in the health sector.

The objective of this book is to provide them with a basic source of information on the use and implementation of standards in clinical and administrative nursing documentation. A compelling case is made for the importance of appropriately documenting nursing care in order to facilitate analyses of nursing activities, quality control, evidence-based direct patient care, and continuity of service. Standardized documentation is also required for communicating nursing concepts, interventions, and outcomes to other nurses and other health professionals working in different settings and countries.

The document focuses on key issues of modern nursing practice and illustrates how information supporting the implementation and use of standard-based practice by means of technology can improve clinical and management tasks in nursing.

http://publications.paho.org • Fax: (301) 206-9789 • Correo electrónico:paho@pmds.com 